\title{
El Programa de Desarrollo y Paz del Magdalena Medio y la Red Prodepaz $^{1}$
}

\author{
María del Rosario Saavedra Andrade
}

\begin{abstract}
The long and complex Colombian conflict has generated, in the different regions of the country, organizations and projects that propose alternatives for a peaceful coexistence and a harmonious development with social inclusion. The Magdalena Medio region has one of the most solid projects of this type. The author of this article shares her experience with the project and presents the main concepts and methodological features that have allowed the sustainability and expansion of this collective effort for several years.
\end{abstract}

\section{Introducción}

La reflexión sobre la intervención social y lo social en la intervención en el contexto actual reviste la mayor importancia para la academia, porque la responsabilidad de la universidad con la formación de las y los jóvenes debe propiciar la posibilidad de reflexionar sobre lo que está sucediendo con los proyectos de carácter social, así como también observar si éstos se reducen a proyectos de corto aliento o si por el contrario se desatan procesos sostenibles. A los esfuerzos de la universidad por acercarse al conocimiento de la realidad y comprometerse con ella, deben sumarse las organizaciones no gubernamentales (ONG), la empresa privada, las iglesias, las organizaciones sociales y populares. Del Gobierno nacional y los gobiernos regionales y locales se espera que no abandonen su responsabilidad

\footnotetext{
${ }^{1}$ Ponencia presentada en el Seminario Internacional sobre la Intervención Social y lo Social en la Intervención, Universidad Icesi, septiembre 28 de 2006.
} 
de ser ejecutores de la intervención social, pues el manejo de lo público le corresponde en primera instancia al Estado Social de Derecho. En esta perspectiva, vale la pena igualmente analizar cuáles son las amenazas y los riesgos de los procesos de intervención social en contextos donde a la sociedad civil se le están delegando, cada vez más, mayores responsabilidades con relación al manejo de los bienes públicos que antes eran competencia del Estado.

En los últimos tres años he estado vinculada desde el Centro de Investigación y Educación Popular (CINEP) con la RED PRODEPAZ y hace dos meses (julio de 2006) terminamos la sistematización de las experiencias que desde su origen (2001) hasta le fecha ha acumulado esta organización como red de redes. He podido hacerlo gracias a mi rol particular como miembro del Comité Operativo de la Red. Me he enriquecido con el análisis colectivo realizado en este espacio sobre los procesos generados por los dieciocho Programas de Desarrollo y Paz que intervienen en zonas de conflicto. ${ }^{2}$ Igualmente tuve la oportunidad de conocer y trabajar en el Magdalena Medio durante cuatro años. En la década de los ochenta y en 2003 participé como miembro del equipo evaluador del segundo préstamo LIL ((Learning and Innovatión Loan - Préstamo de aprendizaje e innovación) del Banco Mundial para el Programa de Desarrollo y Paz del Magdalena Medio desde el Centro de Investigaciones del Desarrollo de la Universidad Nacional. En esta ponencia trabajaré dos puntos: Primero, el origen y evolución del Programa de Desarrollo y Paz del Magdalena Medio (PDPMM). Segundo, los nexos del PDPMM con la Red Prodepaz.

\section{El Programa de Desarrollo y Paz del Magdalena Medio}

\footnotetext{
2 Programa Desarrollo y Paz del Magdalena Medio (PDPMM),Corporación Programa Desarrollo para la Paz del Oriente Antioqueño (Prodepaz), Corporación Nueva Sociedad de la Región Nororiental de Colombia (Consornoc),Corporación Desarrollo para la Paz del Piedemonte Oriental (Cordepaz), Corporación Ambiental Universidad de Antioquia, Programa Darién-Caribe, Secretariado de Pastoral Social Diócesis de San Gil (Sepas), Programa Desarrollo y Paz del Suroeste Antioqueño ,Justapaz -Programa Construcción de una Infraestructura para la Paz y la Convivencia Democrática a partir de los Montes de María hoy Fundación para el Desarrollo y la Paz de los Montes de María, Fundación Pro-Sierra Nevada de Santa Marta, Programa de Paz y Competitividad del Eje Cafetero, Corporación para el Desarrollo y Paz del Valle (Vallenpaz),Consejo Regional Indígena del Cauca (CRIC) y Asopatía, Programa una “Mirada al Sur”, Fundación El Alcaraván, Programa de Desarrollo y Paz del Tolima Tolipaz, Grupo Gestor para el Desarrollo de Casanare, Programa de Desarrollo y Paz del Magdalena Centro, Programa de Desarrollo y Paz del Huila (Huipaz).
} 
En medio de la guerra que vive Colombia, quienes llevamos muchos años haciendo intervención social podemos caer fácilmente en la desesperanza. El contexto colombiano muestra signos de profundización de la exclusión y la pobreza - 50\% de las y los colombianos están en situación de pobreza - a pesar de que los indicadores de la macroeconomía muestren cifras de crecimiento del 6\%. El fenómeno social del desplazamiento masivo como consecuencia de la guerra se constituye en una de las más graves crisis humanitarias del planeta. Actualmente hay alrededor de dos millones de colombianos y colombianas desplazadas de manera forzada por el conflicto armado. La desigualdad y la falta de oportunidades para los jóvenes son alarmantes y son caldo de cultivo para que ellos y ellas se vinculen desde muy temprana edad a los grupos armados, pues la sociedad no les ofrece oportunidades ni les garantiza los derechos que están consagrados en la Constitución.

Este diagnóstico sombrío contrasta sin embargo con la realidad de que el país cuenta con una reserva ética y moral de hombres y mujeres que le están apostando al futuro por una sociedad donde los conflictos se puedan tramitar pacíficamente por la vía política y donde todos y todas quepamos sin exclusiones. Un caso emblemático es el Programa de Desarrollo y Paz del Magdalena Medio (PDPMM), creado en una de las regiones de Colombia caracterizada históricamente por la mayor resistencia social, obrera, campesina, y popular. En el Magdalena Medio las mujeres de la Organización Femenina Popular llevan 34 años de historia de lucha y reconocimiento nacional e internacional y la Iglesia Católica ha intervenido con una visión abierta y comprometida con los pobres. Durante años los jesuitas han tenido allí una de sus misiones más destacadas. El director del PDPMM Francisco de Roux, jesuita, fue director del CINEP por siete años y maduró su experiencia de más de 20 años de intervención social desde su trabajo comprometido con los sectores populares urbanos de Bogotá en los años setenta. Calificamos de emblemático al PDPMM, a pesar de que su formulación data de 1995 y hay programas en la Red Prodepaz de mayor antigüedad, porque el enfoque metodológico que propone el PDMMM de intervenir y buscar el desarrollo en medio del conflicto ya y ahora tiene un carácter pionero. 
Fueron las condiciones de violencia y pobreza del Magdalena Medio, y la precaria presencia e incapacidad del Estado para resolver esta problemática, las que le dieron origen al PDPMM a mediados de 1994. En su conformación participaron el Comité de Derechos Humanos de la Unión Sindical Obrera USO-ECOPETROL, la Pastoral Social y la diócesis de Barrancabermeja y la Sociedad Económica de Amigos del País (SEAP). La creación del PDPMM fue encomendada al consorcio SEAP-CINEP, que fue el gestor del programa entre 1996 y 1998. El proceso que ha generado el PDPMM no ha sido lineal. Por el contrario ha tenido altibajos y ha sido un proceso de ajuste permanente, de búsqueda por salvar la consistencia. Si no lo hubiera hecho así, habría quizás perdido la participación de organizaciones sociales y sindicales fuertes, tales como la Unión Sindical Obrera, la OFP, o los pescadores organizados. Igualmente ha tenido que concertar permanentemente con el Gobierno nacional, con los gobiernos locales, con la cooperación internacional, construyendo la autonomía a diario y luchando para que su propuesta se mantenga. Todo esto igualmente a pesar de los actores armados que han intentado sabotearla muchas veces y le han quitado la vida a más de 20 personas que han estado vinculadas al Programa. De ahí su complejidad.

\section{Intervención en la región}

Después de realizar un cuidadoso diagnóstico de la región (De Roux, 1996), el Programa se fijó como objetivos:

a) Ampliar el control de la comunidad sobre los excedentes que genera la región. El desarrollo económico debe ir en consonancia con un mejoramiento de la dignidad y calidad de vida.

b) Propender por la sostenibilidad ecológica, económica y social como elementos centrales de la estrategia.

c) Construir una democracia participativa y una consolidación del espacio de lo público. El análisis de fondo que sustenta esta ruta es que no es posible que una región tan rica como la del Magdalena Medio (por sus habitantes, el petróleo, las minas de oro, la biodiversidad,) pueda vivir con municipios y veredas en donde la pobreza alcanza niveles muy altos. 
Tampoco es posible que un pueblo alegre, trabajador, soñador, viva una situación de tanta violencia.

En 1997 el PDPMM comienza con la creación de los núcleos municipales ${ }^{3}$ y la propuesta municipal. Desde el comienzo el Programa fue consciente de que no podía ser una mera agencia financiadora de proyectos. La etapa de innovación y aprendizaje se fue concretando gracias al crédito denominado LIL (Learning and Innovatión Loan) del Banco Mundial a la Nación. El Banco participa con USD \$ 5 millones y ECOPETROL con una contrapartida de USD \$ 1.1 millones.

En 1998 se creo el Consorcio de Desarrollo y Paz del Magdalena Medio (CDPMM), constituido por la Diócesis de Barrancabermeja y el Centro de Investigación y Educación Popular (CINEP), como una entidad independiente y autónoma que administraría y ejecutaría los recursos del crédito. En esta nueva etapa, que duró hasta el año 2000, se buscó generar procesos de aprendizaje y ampliación de las capacidades de los distintos actores sociales, locales, regionales, públicos o privados. El Programa pretende que a partir de la identificación de las necesidades y problemáticas, los mismos pobladores sean quienes formulen propuestas y proyectos de impacto e interés municipal y regional. Las prioridades deben ser definidas por la comunidad implicada. A través de este proceso se trata de hacer realidad la metodología de la participación municipal y regional.

En el año 2001, el Gobierno colombiano y el Banco Internacional de Reconstrucción y Fomento (BIRF-Banco Mundial) negociaron la financiación de una segunda fase del préstamo LIL. La entidad ejecutora fue el Departamento Nacional de Planeación (DNP), que delegó la implementación del proyecto a la Corporación Desarrollo y Paz del Magdalena Medio (CDPMM) y la administración de los recursos al Programa de las Naciones Unidas para el Desarrollo (PNUD). ${ }^{4}$ El préstamo del BIRF fue de USD \$5.000.000, con una contrapartida de USD \$1.168.034 del Gobierno nacional, representada en los aportes de

\footnotetext{
${ }^{3}$ El núcleo, como su nombre lo indica, es el centro de construcción de lo público. A través del núcleo se busca propiciar un ambiente de paz y de convivencia. Las propuestas municipales son la expresión de las necesidades captadas por el núcleo. El papel estratégico del núcleo se puede mantener si su campo de acción se amplia y no se limita a los beneficiaros de los proyectos productivos.

${ }^{4}$ Programa de las Naciones Unidas para el Desarrollo, Proyecto del Gobierno Nacional. Proyecto de Aprendizaje e Innovación para el Programa de Desarrollo y Paz del Magdalena Medio - Fase II.
} 
ECOPETROL, y una contribución de USD \$175.000 de la Agencia Colombiana de Cooperación Internacional (ACCI) y el PNUD. El valor total fue de USD \$6.343.034.

La dinámica que adquirió el Programa de Desarrollo y Paz obligó a crear una entidad con la capacidad y estructura administrativa y financiera para manejar y asumir los compromisos exigidos por el crédito LIL II. El Consorcio de Desarrollo y Paz del Magdalena Medio se transformó entonces en Corporación. De la nueva institución hicieron parte el Centro de Investigación y Educación Popular (CINEP) y la Diócesis de Barrancabermeja, que ya hacían parte del Consorcio. La corporación tiene mayor libertad de gestión y administración que el consorcio. Puede permitir el ingreso de otros asociados y tiene la potestad de recibir y administrar aportes y ayudas de origen nacional e internacional. En esta segunda fase de Aprendizaje e Innovación fue evidente el esfuerzo para que los pobladores se convirtieran en los principales actores del PDPMM. Se insistió en la necesidad de potenciar las capacidades de los pobladores de tal forma que pudieran adquirir responsabilidades en el manejo y orientación del PDPMM.

La segunda fase del LIL II tuvo cuatro componentes:

a) Organizacional (institucional y organizativo). Busca mejorar las capacidades y el margen de gestión de las organizaciones de los diferentes municipios de la región. Son los Equipos Técnicos Pedagógicos (ETP) ${ }^{5}$, el Equipo de Coordinación Regional (ECOR), ${ }^{6}$ y los Núcleos municipales las instituciones rectoras de este proceso.

b) Fortalecimiento institucional. Desarrolla instrumentos para que las instituciones locales y regionales, públicas y privadas, respondan a los intereses de la región. Se trata de que los Núcleos de pobladores participen en las políticas, planes y programas de las administraciones municipales a través de la Propuesta Municipal.

c) Económico productivo. Apoya las actividades económicas productivas en diversas áreas (tecnología, insumos, comercialización, capacidad empresarial y financiamiento). Se ponen

\footnotetext{
${ }^{5}$ Los ETP (Equipos Técnicos Pedagógicos) son el punto de encuentro y retroalimentación entre las directrices políticas y administrativas generales de la CDPMM y los procesos concretos en las subregiones y municipios, de tal modo que se hagan efectivas las estrategias de desarrollo y paz. Los miembros de los ETP son cercanos a los núcleos de pobladores y las subregiones.

${ }^{6}$ Equipo de Coordinación Estratégica, ya no existe.
} 
en práctica proyectos como finca campesina, desarrollo agroindustrial, micro regiones y ecosistemas estratégicos, y la economía de pueblos.

d) Social. Tiene que ver con el fortalecimiento de iniciativas sociales con las personas más vulnerables por la pobreza y por el conflicto armado.

\section{Reflexiones sobre los alcances de la intervención social del PDPMM}

En la dinámica regional del Magdalena Medio confluyen fuerzas heterogéneas y de muy diversa naturaleza, y uno de estos actores es el Programa de Desarrollo y Paz del Magdalena Medio. Es muy difícil establecer las fronteras de los impactos (directos e indirectos) generados por el Programa y los producidos por otros actores y factores que también están afectando o contribuyendo con el desarrollo regional. El PDPMM propone un modelo de desarrollo alternativo a la guerra en un contexto social muy complejo. La propuesta del Programa va ganando fuerza en la medida en que forma parte de la organización institucional de la región (González, Castilla y Merchán, 2003; García y Sarmiento 2002). El PDPMM hace una propuesta de desarrollo regional que nos parece adecuada porque supera el esquema fiscalista que ha caracterizado el proceso de descentralización en Colombia. El Programa asume seriamente un enfoque de desarrollo regional y reconoce de manera explícita las virtudes que se derivan de los procesos endógenos virtuosos. Y aunque la Constitución de 1991 le da una importancia central al desarrollo regional, en el país no se ha avanzado en la reglamentación de los normas de ordenamiento territorial. La regulación continúa discutiéndose y aún no se concreta en una ley.

El hecho de mantener el Programa de Desarrollo y Paz en medio de un contexto de violencia y conflicto armado es, de por sí, un valor agregado para la región y el país. Los valores que promueve (la paz, la vida, la productividad, la aceptación de la diferencia y la solidaridad), y las capacidades que intenta desarrollar en los pobladores (asociación, iniciativa organizativa, capacidades para la toma de decisiones que los afecten, etc.) tienen una bondad intrínseca. El Programa ha logrado posicionarse en la región e intervenir de 
manera positiva y alternativa. Ha contribuido a generar importantes espacios de convivencia, tolerancia y fortalecimiento del capital social y el desarrollo humano.

La permanencia y el compromiso adquirido y demostrado por los trabajadores y trabajadoras de la Corporación y del PDPMM refleja un espíritu de gratuidad que va más mucho allá del contrato laboral. Para algunos la vinculación al PDPMM se ha convertido en una verdadera opción de vida. Esta es una labor y una actitud que merece el mayor reconocimiento, dadas las condiciones de riesgo y amenaza en que desarrollan sus tareas. Este espíritu de trabajo y solidaridad contribuye a la construcción del capital social y al desarrollo regional. Los beneficiarios de los proyectos confían en el Programa, tanto por su pertinencia como por la transparencia en el manejo de los recursos públicos. En las entrevistas observamos que aún las personas que más critican al Programa reconocen que la administración de recursos es transparente.

Como evaluadores del segundo préstamo LIL afirmamos en 2003 que, según nuestra apreciación general, la metodología del LIL I y LIL II ha tenido buenos resultados, en el sentido de que ha fortalecido procesos sociales y organizativos y ha permitido afianzar aprendizajes e innovaciones. La metodología tiene una mirada integral que comprende las formas de exclusión económica, política y social que son muy acentuadas en el Magdalena Medio. El PDPMM ha logrado “navegar” de manera exitosa en medio del conflicto sociopolítico de la región. El Programa ha realizado un trabajo de filigrana política admirable, que le ha permitido sacar adelante diferentes iniciativas de los núcleos municipales. El Programa ha ganado respeto y confianza en los niveles regional, nacional e internacional. La transparencia en el manejo del dinero es uno de los aspectos que más destacan las personas entrevistadas. Sin embargo, con el monto de recursos que administra la Corporación no es posible financiar un proceso de desarrollo regional que transforme de manera radical las estructuras económicas existentes. En este sentido, el Programa es uno más entre los actores que participan en la región. En la formulación de los objetivos del Programa se sobredimensionan las posibilidades de la intervención, pues no está en manos del PDPMM la transformación de la región. A pesar de que esta apreciación sea relativamente obvia, sorprende que alrededor del Programa soplen vientos que intentan dejar 
en sus manos todo el desarrollo regional. Esto se debe a que el Gobierno nacional y las entidades financiadoras han puesto sobre los hombros del Programa una tarea de dimensiones titánicas. Las actividades productivas que realiza el Programa son complementarias a las dinámicas económicas públicas (ECOPETROL) y privadas (ganadería extensiva y cultivos de palma y cacao). Un proyecto de desarrollo regional que tenga como objetivo la transformación de la región del Magdalena Medio requiere de mucho tiempo y fuertes recursos. El PDPMM cuenta con el tiempo, toda vez que sus acciones se han proyectado inicialmente a quince años. También cuenta con recursos de inversión para mantener su presencia de manera sostenida. Sin embargo es importante resaltar que un proyecto de desarrollo regional implica una gran inversión tanto del Estado como de la empresa privada. Para ilustrar esta afirmación basta con sumar el costo de los principales proyectos de infraestructura urbana y regional: Troncal de la Paz, plan maestro de acueducto y alcantarillado de Barrancabermeja, terminal de transporte de Barrancabermeja, vías de conexión regional, puente de Yondó. ${ }^{7}$

La riqueza de la propuesta del PDPMM debe mirarse mas allá de las transformaciones económicas que logre a través de sus proyectos productivos, y más bien debe verse como portador de una dimensión profética de las acciones que realiza. El Programa está mostrando que en medio del conflicto es posible construir una sociedad distinta, basada en la inclusión. Y el préstamo LIL II contribuyó positivamente a lograr este propósito. Ahora el Programa tiene el enorme reto de lograr que su propuesta impregne la dinámica institucional local de tal manera que genere procesos endógenos virtuosos. Desde 2003 hasta la fecha, se ha avanzado significativamente en éste sentido. El proceso de consolidación de las relaciones interinstitucional ha sido diferenciado. El LIL II consolidó algunos procesos de los núcleos tendientes a la construcción de la visión municipal y regional. Observamos que se ha fortalecido un espacio para el diálogo y la concertación en los municipios. En líneas generales, podría decirse que en los municipios de influencia del Programa se han creado condiciones favorables al debate público. En algunas subregiones se logró un aumento

\footnotetext{
${ }^{7}$ El plan maestro de acueducto y alcantarillado de Barrancabermeja tiene un valor aproximado de 23 mil millones de pesos, y el terminal de transporte de Barrancabermeja puede ascender a 15 mil millones de pesos.
} 
significativo de las capacidades, y se constata que los pobladores han mejorado sus competencias para definir e implementar proyectos.

\section{EI PDPMM y la recuperación del sentido de lo público}

En varias administraciones municipales se aprecia que LIL II fue un escenario de avance importante en las relaciones del orden nacional (el Sena, la Red de Solidaridad, el Ministerio de Agricultura, FINAGRO, ECOPETROL), regional (Cormagdalena, las gobernaciones de la región del Magdalena Medio, las secretarías de salud y educación) y municipal (Umatas, alcaldías, cámaras de comercio de Barrancabermeja y Aguachica, Fedepalma y Fedecacao, la USO, Defensoría del Pueblo), que se constituyeron en espacios de la agenda semanal del Programa en el proceso de recuperar la acción pública para lo público. Fue en el espíritu del Marco Lógico que, al preparar el Laboratorio de Paz como continuación del LIL y en plena vigencia de éste, se crearon las asambleas municipales y los comités municipales (ad hoc), presididos por los alcaldes, para definir los proyectos del Laboratorio de Paz I. Así, el avance en la articulación entre el PDPMM y las alcaldías municipales se ha dado gracias a los lazos de confianza que se han construido.

Jorge Iván González (2003), refiriéndose al PDPMM, afirma lo siguiente:

Creo que el gran mérito del PDPMM es decir "sí es posible el desarrollo ya y en medio de unas situaciones muy complejas”. Esa idea no está inscrita en la construcción del desarrollo económico colombiano y eso es desastroso, porque si nosotros tenemos que esperar con sudor y lágrimas, como lo señala el FMI y lo asume el gobierno colombiano, a que se dé la inclusión en una etapa posterior y la gran preocupación es el desequilibrio fiscal, entonces se ahonda la desigualdad y la exclusión y se aleja la paz. Empezar desde ya es la diferencia fundamental entre dos esquemas de desarrollo, y empezar a pensar seriamente en una agenda distributiva es un tema que no se puede postergar más. ${ }^{8}$

Se debe entender, siguiendo la perspectiva de Amartya Sen, que las variables macroeconómicas son herramientas para alcanzar la libertad, la cual se basa, al mismo

\footnotetext{
${ }^{8}$ Jorge Iván González, Conversatorio, Redprodepaz, Bogotá, 2003.
} 
tiempo, en instituciones sociales y políticas que faciliten la educación, la salud, la participación y el debate. En ese momento se puede hablar de desarrollo, pero no cuando se toma en cuenta solamente el producto per capita de una nación o sus ingresos. Esta forma de entender el desarrollo implica, de manera decisiva, la inserción de un nuevo sistema de valores en el cual sea preponderante la responsabilidad ética, la solidaridad y el cuidado del bien común y del espacio público. Se requiere un cambio de la perspectiva egoísta e individualista de la sociedad de consumo por una concepción de desarrollo humano incluyente y sostenible, de solidaridad, para lo cual la autonomía de las organizaciones y de las personas, así como la vida en armonía con la naturaleza, son un requisito. El desarrollo tal como se concibe en el PDPMM tiene un componente moral que implica que lo público se conciba como un bien que pertenece a la comunidad y no como un bien enajenado por un grupo de individuos particulares. Lo público pasa a ser, entonces, la institucionalización del bien colectivo, razón por la cual no se lo puede dejar en manos de unos pocos sino que debe tener características participativas en su construcción. En esa medida se considera la diferencia entre lo público, lo privado y lo estatal como escenarios de interacción diferentes.

En cuanto a lo público, compartimos las definiciones de lo público, lo estatal y lo privado que se proponen en el documento de evaluación del Fondo para la Reconstrucción del Eje Cafetero (FOREC) realizado por el Centro de Investigaciones del Desarrollo (CID, 2003) de la Universidad Nacional. Lo público cubre el espacio atravesado por la elección colectiva. Esta elección tiene que ver con la elección entre estados del mundo alternativos. Hay muy distintas formas de elección colectiva y su diversidad depende del ámbito en que se llevan a cabo. El paso de la elección individual a la colectiva no se presenta exclusivamente en el campo político. En realidad, permea todas las esferas de la vida social en las que tiene lugar, de forma implícita o explícita, una elección colectiva. Hay elección colectiva en la familia, la empresa, el colegio, la universidad, la iglesia, el pueblo de mil habitantes, la ciudad de 15 millones. Mientras que en las primeras (familia, empresa, Iglesia católica) tiende a predominar la imposición, en el campo político puede dársele prioridad a la regla de decisión por mayoría. ${ }^{9}$ Lo estatal se refiere a las formas de regulación y de apropiación de

\footnotetext{
9 Jorge Iván González, "El FOREC como modelo de intervención del Estado", Universidad Nacional de Colombia, Red de Solidaridad, CID, 2003, (p. 16)
} 
los bienes mediados por la elección colectiva. Lo privado tiene que ver con los bienes que no pasan por el tamiz de la elección colectiva. Siempre que se presente alguna forma de elección colectiva lo público hace presencia. El paso de la elección individual a la colectiva puede llevarse a cabo de muchas maneras: regla de decisión por mayoría, imposición, dictadura, unanimidad, etc. Hay expresiones de lo público en las que predomina la imposición, y los fines que se proponen como favorables a todos únicamente sirven a los intereses de quienes tienen la capacidad de imponerlos (González, 2003).

En los últimos años se ha insistido en la importancia de que las ONG participen en la ejecución de programas gubernamentales. Se ha dicho, incluso, que es más eficiente el manejo del gasto cuando su ejecución es delegada a las ONG que cuando la realiza directamente el Estado. ${ }^{10}$ Para analizar el modelo de intervención debe tenerse en cuenta en primera instancia el sentido de lo público y, en segunda, la experiencia de la respectiva ONG.

Una de las preocupaciones permanentes del PDPMM ha sido el estímulo a la participación comunitaria en el manejo de lo público. La ampliación del sentido de lo público favorece la inclusión y la paz. ${ }^{11}$ Para que la participación sea efectiva es fundamental que se refleje en acciones concretas. No basta con opinar, es necesario transformar la opinión en logros específicos. Desde el comienzo el PDPMM expresó su voluntad de trabajar con los políticos y con los poderes locales. ${ }^{12}$ Hay dos razones por las

\footnotetext{
${ }^{10}$ El Fondo de Reconstrucción del Eje Cafetero (FOREC) se presentó no sólo como un modelo exitoso, sino como el nuevo tipo de Estado. Ver, además, CID (2003), Crece (2002), Cuervo (2001, 2002), Gómez, Castaño y Duque (2002), González (1999), Sánchez, Cendales y Mariño (2001), Saavedra y Albán (2002).

11 "El PDPMM tiene como intención la construcción de lo público en el Magdalena Medio, como un requisito para el logro de la convivencia pacífica, la tolerancia y el alcance de una paz digna. La materialización de esta intención debe enfrentarse, a una elaboración conceptual que permita llenar de contenido y de sentido "lo público", y un análisis y comprensión de un escenario histórico particular como es la región de intervención del Programa” (González, Jorge Iván, “Imaginarios de Desarrollo y Paz en el Magdalena Medio”, CINEP, 2003)

${ }^{12}$ Ibíd. Esta actitud es muy distinta a la que tuvo el FOREC, que siempre trató de desconocer a los políticos locales. El Centro Interdisciplinario de Estudios Regionales (CIDER, U. de los Andes) observa que, en contra de la voluntad de PDPMM, la relación con las instancias políticas de la zona no ha sido fácil. "Desde el momento mismo de su concepción, se consideró que el Programa era un instrumento de desarrollo y paz que no estaba llamado a sustituir la acción de las entidades públicas y particularmente de los municipios, pero sí a trabajar en estrecha armonía con éstos. No obstante, por razones de diversa índole, algunas de las cuales fueron examinadas en el curso de la Primera Fase de la Evaluación Externa, estas relaciones no han sido tan constructivas como hubiera sido deseable" (CIDER, 2000: 59). Como lo reconoce el CIDER, la situación cambia mucho de un municipio a otro. Y en algunos casos la heterogeneidad del panorama está muy marcada por la influencia que ejercen los grupos armados en las autoridades locales.
} 
cuales la opción del PDPMM es correcta. En primer lugar, porque los gobernantes locales han sido escogidos mediante la regla de decisión por mayoría, y este procedimiento para pasar de la elección individual a la elección colectiva es aceptado como legítimo por las democracias contemporáneas; en segundo lugar, porque el Programa únicamente es sostenible en el mediano y largo plazo si se integra a la institucionalidad vigente en la región.

La práctica previa y el conocimiento que tengan las ONG son fundamentales para garantizar el éxito de los programas que buscan delegarles la administración y la ejecución de los recursos públicos. En el momento en que CINEP participó en la fundación del PDPMM ya tenía una experiencia de 25 años. Y el aprendizaje previo de las ONG es más importante si el propósito de la intervención es complejo. ${ }^{13}$ Nos referimos no sólo a la preparación técnica. También es fundamental la percepción que la ONG tenga de lo público. Por ejemplo, el PDPMM entendió las potencialidades que tiene la organización institucional de la Iglesia, y por ello la vinculó al Programa desde el principio. ${ }^{14}$

\section{El concepto de "región”}

La conceptualización de “región” se está dando más en el terreno de la experiencia práctica, como es el caso del PDMM. En este programa la región imaginada es aquella en la cual los procesos de descentralización intentan ser amplios intentos regionales de descentralización espacial que no se reducen a los proyectos de descentralización fiscal y administrativa. Es posible definir a la región conforme a parámetros identitarios partiendo de las relaciones establecidas entre los pobladores y al mismo tiempo entre éstos y su entorno. La región se entiende como una construcción que se erige sobre la base de las expresiones territoriales concretas de sus pobladores. Como lo expresa el director del PDPMM:

Uno se vincula desde la identidad regional que ayuda a colocarlo en la globalización alternativa, porque se vincula no desde la abstracción del

\footnotetext{
${ }^{13}$ La construcción de casas es un objetivo menos complejo y más concreto que la definición de alternativas viables para la paz. Los ritmos de ejecución del FOREC fueron buenos, entre otras razones, porque SU objetivo era claro.

${ }^{14}$ González, Jorge Iván, Op. Cit.
} 
Estado-nación sino que uno se vincula étnicamente, culturalmente, espiritualmente e incluso económicamente desde unidades regionales que son más concretas. Se dan procesos de hermandad sin que necesariamente se deba estar sujeto a la división político administrativa del país. ${ }^{15}$

Es de esta forma como se entiende que la región es una construcción cultural que brota de lazos identitarios presentes en una historia conjunta y un ambiente compartido y que determina así relaciones específicas entre las personas y el entorno, que son diferentes de las de otros lugares del país. Sólo de esta forma se entiende, por ejemplo, la construcción de la región del Magdalena Medio, que encuentra su punto de articulación en el petróleo y el río Magdalena, lo cual ha originado rasgos característicos propios en los que confluyen diversos departamentos.

Uno de los grandes obstáculos para la consolidación de las regiones como entes jurídicos es la inexistencia de una definición legal, debido a la carencia de un plan de ordenamiento territorial nacional que debería ser de carácter participativo para que pudieran verse reflejados los lazos identitarios. Esta indefinición ha traído como consecuencia que el término región, por no tener correspondencia jurídica en el país, sea ambiguo y no se refleje en la administración política. Pero igualmente la ausencia del ordenamiento territorial se plantea como un obstáculo para el desarrollo de los Programas de Desarrollo y Paz puesto que las regiones expresadas por los habitantes no coinciden con la división política ratificada por la Constitución de 1991. De ahí que cuando se alude a la construcción de nación con base en las regiones se hace referencia a parámetros sin correspondencia con el ordenamiento político-administrativo del país. En este sentido, la evaluación externa del PDPMM (2003) dice lo siguiente:

Aunque la Constitución de 1991 le da una importancia central al desarrollo regional, en el país no se ha avanzado en la reglamentación de las normas de ordenamiento territorial. La regulación continúa discutiéndose y aún no se concreta en una ley. Con los hechos, el PDPMM también está demostrando que es fundamental replantear la descentralización de naturaleza fiscal y volver la mirada hacia las regiones. El mercado interno, que es un elemento central del proyecto regional, no avanza porque la infraestructura vial es muy débil. A diferencia de otros países, Colombia acabó con el transporte fluvial y férreo. Y el sistema de carreteras es de muy mala calidad. Estos problemas de

\footnotetext{
${ }^{15}$ Entrevista al director del PDPMM, Barrancabermeja, octubre de 2004.
} 
infraestructura obstaculizan la expansión de los circuitos económicos regionales y nacionales. En la perspectiva de largo plazo, por ejemplo, el Programa de Desarrollo y Paz del Magdalena Medio es consciente de la necesidad de consolidar los circuitos productivos internos y los encadenamientos intersectoriales. ${ }^{16}$

\section{Trabajo en red}

El inicio de la Red Prodepaz aparece ligado a dos contextos. Primero, el reconocimiento del gobierno nacional y la cooperación internacional del papel que desempeña la sociedad civil en la construcción del desarrollo y la paz a partir del ejemplo del PDPMM. Segundo, la necesidad de ampliar la experiencia articulándose con otras experiencias regionales a través de una red. En el año 2001 se reunieron en San Gil (Santander) directores y representantes de 14 iniciativas y programas ${ }^{17}$ con las instituciones de apoyo a los procesos regionales, ${ }^{18} \mathrm{y}$ emitieron la declaración de principios "Por una Colombia en paz construida desde las regiones”, y a comienzos de 2002 los programas regionales ya articulados en una red definieron su meta social y económica en pro de un desarrollo humano integral:

Nuestra propuesta tiene una voluntad espiritual y social inquebrantable (...) Trabajamos por superar la exclusión y la violación de los derechos humanos y para asumir con determinación nuestros deberes ciudadanos. Pero, al mismo tiempo, nuestra propuesta se presenta como una alternativa técnicamente viable y sostenible (...) Estamos construyendo en cada región un plan de desarrollo humano integral y de paz con grandeza. ${ }^{19}$

\footnotetext{
${ }^{16}$ Evaluación externa al PDPMM, pág. 3.

${ }^{17}$ Programa Desarrollo y Paz del Magdalena Medio (PDPMM), Corporación Programa Desarrollo para la Paz del Oriente Antioqueño (Prodepaz), Corporación Nueva Sociedad de la Región Nororiental de Colombia (CONSORNOC),Corporación Desarrollo para la Paz del Piedemonte Oriental (Cordepaz), Corporación Ambiental Universidad de Antioquia, Programa Darién-Caribe, Secretariado de Pastoral Social Diócesis de San Gil (SEPAS), Programa Desarrollo y Paz del Suroeste Antioqueño (Justapaz), Programa Construcción de una Infraestructura para la Paz y la Convivencia Democrática a partir de los Montes de María , Fundación ProSierra Nevada de Santa Marta, Programa de Paz y Competitividad del Eje Cafetero, Corporación para el Desarrollo y Paz del Valle (Vallenpaz),Consejo Regional Indígena del Cauca (CRIC),Programa una "Mirada al Sur, Fundación El Alcaraván. A éstas 14 iniciativas y programas de desarrollo y paz se han sumado otras como: Programa de Desarrollo y Paz del Magdalena Centro, Programa de Desarrollo y Paz del Huila (Huipaz), Grupo Gestor para el Desarrollo de Casanare.

${ }^{18}$ Interconexión Eléctrica ISA, CINEP, Fundación Social.

${ }^{19}$ Manifiesto por una Colombia en paz construida desde las comunidades locales y desde las regiones
} 
En el encuentro de San Gil las iniciativas y programas de desarrollo y paz propusieron funcionar en red. Se produjeron contactos espontáneos y naturales entre personas animadas por la secretaría ad hoc que asumió Interconexión Eléctrica ISA durante el primer año de funcionamiento. Fue un año de consolidación de confianzas, de interacción y de solidaridad, de aumento del capital social. Según el representante de ISA, la Red Prodepaz se remonta en su origen a una conferencia del director del PDPMM dictada a finales de 1998 en ISA S.A, en la cual se manifestó claramente una perspectiva de trabajo a partir de tres grandes ejes: la perspectiva regional, la acción interinstitucional y una visión de largo plazo que supere los episodios electorales de cada periodo.

Posteriormente, se le pidió al director del PDPMM que, a partir de la reflexión generada por el primer documento de declaración de principios, se hiciera una nueva propuesta para presentarla a los candidatos presidenciales en las elecciones de 2002, con el ánimo de darle continuidad al proceso y enviarles el mensaje de que los programas de desarrollo y paz (PDP) seguirían con ellos o sin ellos. Era importante que consideraran la magnitud y la relevancia del proceso y que comprendieran la propuesta que encarnaba:

En marzo de 2002 se suscribió la declaración y se tuvo una reunión con la Dirección Nacional de Planeación DNP, donde se sentaron unos principios básicos de los programas, cuyo sentido era: aquí no podemos competir entre los PDP. Cada uno haciendo gestión por su lado es débil, y cada uno debilita a los otros por querer acceder a los recursos por su cuenta. Aquí tenemos que ir todos en conjunto con un pensamiento sólido que le dé fuerza al proceso y donde hagamos una gestión que nos beneficie a todos. Era un planteamiento muy claro y central de los programas y era una garantía de que todos los procesos actuaban de una manera conjunta. Los planteamientos filosóficos y los principios de los PDP le daban una coherencia al trabajo en red: estamos construyendo una región en paz desde estos procesos locales y regionales. Y tenemos la determinación de seguir adelante con o sin gobierno o sin cooperación internacional. Haciendo todos los esfuerzos para que la gente permanezca en su sitio de origen, y siempre de acuerdo con unos principios fundamentales de la red y unos objetivos claros. El año 2002 fue un año de mucho trabajo, donde se logró hacer un pensamiento estratégico. Lo que hoy aparece en la red como ejes de actuación, principios objetivos y visión, se construyó en $2002{ }^{20}$

\footnotetext{
${ }^{20}$ Entrevista a representante de ISA, Bogotá, octubre de 2005.
} 


\section{Caracterización de la Red Prodepaz}

Caracterizar a la Red Prodepaz es tarea compleja porque, como se dijo arriba, los PDP que trabajan en red son heterogéneos, los territorios y contextos culturales donde se mueven son diversos, los intereses de quienes los conforman son distintos. Algunos PDP tienen una fuerte influencia de las universidades, como el Programa de Paz y Competitividad del Eje Cafetero que ha sido impulsado por la Universidad Autónoma de Manizales. Otros han sido “jalonados” por empresarios, como es el caso de Vallenpaz. Otros como CRIC-Asopatía han sido promovidos por la Asociación de Municipios del Patía y el Consejo Regional Indígena del Cauca. Otros, como Prodepaz, han sido impulsados por la Iglesia Católica e Isa. En casi todos los PDP las universidades están presentes: en CONSORNOC, la Universidad de Pamplona; en Sepas, la Universidad de San Gil; en el Magdalena Medio, la Universidad de la Paz; Darién Caribe ha sido impulsado por la Universidad de Antioquia; Tolipaz, por la Fundación Social y la Iglesia del Tolima; Mirada al Sur, por CINEP y la Fundación Social; la fundación para el desarrollo y paz de los Montes de María ha sido impulsada por la Iglesia Católica y la Iglesia Menonita. Como vemos la composición de los PDP es heterogénea.

Por otro lado, se presenta una dificultad cuando se intenta caracterizar a los programas como miembros de la "sociedad civil", porque el término en sí mismo es ambiguo. En la página web de la Red encontramos que su naturaleza se define como "un sistema de coordinación de la sociedad civil, plural y autónomo, que convoca y articula a Programas Regionales de Desarrollo Integral y Paz. Este sistema está constituido por un conjunto de entidades que comparten el interés común de construir una nación en paz a través de la participación social y comunitaria en procesos locales y regionales de desarrollo humano, integral, sostenible, equitativo y solidario”. Sin embargo, el término sociedad civil, en sí mismo, es poco claro. Sergio Bossier se refiere así a este término:

Personas y organizaciones de personas que poseen una visión colectiva de sí, la que, aunque difusa, es cohesionada por la participación y por la movilización de las y los actores en torno a la consecución de objetivos relativamente autónomos respecto del Estado, de la actividad política profesional y del mercado, pues si 
bien la sociedad civil no pretende hacerse del control de estas entidades, ella no es absolutamente independiente y extraña a aquellas (sic). El éxito de la sociedad civil podrá medirse en su grado de influencia, adopción y proyección en el Estado, las instituciones políticas y el mercado. Por eso, los temas de la sociedad civil van desde las relaciones de las personas entre sí y su cotidianidad, hasta las organizaciones requeridas por las precauciones necesarias y expresiones nacidas de los intereses de la vida en común. Por ello es que la sociedad civil es el amplio espacio articulador entre lo privado y lo público. ${ }^{21}$

Puede decirse que lo que caracteriza la pertenencia a la Red Prodepaz es la actividad en redes regionales y locales, donde participan individuos, grupos y organizaciones que trabajan en regiones en conflicto, porque en cada lugar en donde está presente un PDP o una iniciativa, su configuración y su modo de acción (intervención) responde al trabajo en red y al propósito de tejer relaciones de confianza. Por eso, la Red Prodepaz es considerada una red de redes, un proceso en construcción y validación que busca articular un espacio para los programas de desarrollo y paz en regiones que soportan un conflicto intenso y prolongado. Es un espacio de encuentro y de intercambio de aprendizajes y experiencias, de información, ajuste y colaboración, coordinación, establecimiento de relaciones e incremento de confianza en la forma como se involucran y en el respaldo que se dan. Los miembros fundadores de la Red perciben que el punto de articulación de los PDP son los derechos humanos, porque el desarrollo es un derecho humano y debe servir para el bienestar individual y colectivo y para el disfrute de las personas, sin exclusiones. Siguiendo a un autor como Van Deventer, puede decirse que la Red Prodepaz y sus miembros que trabajan en red

(...) se diferencian de las organizaciones intermediarias tradicionales en muchos aspectos. Son más difíciles de definir como unidad o entidad, ya que se componen de un grupo de entidades que formal o informalmente están relacionadas entre sí. Estas entidades, los miembros o participantes de las redes, pueden ser personas a título individual, partes de una organización, organizaciones individuales o incluso redes. Participar en una red parece un proceso voluntario y racional en el cual tanto el participante como la red esperan beneficiarse de esta relación. ${ }^{22}$

\footnotetext{
${ }^{21}$ Bossier, Sergio, “Crisis y alternativas en los procesos de regionalización”, en: Revista de la CEPAL, No. 52,
} abril 1994, (p. 3) 
En el caso de la Red Prodepaz, sus miembros participan más allá de su compromiso individual, por una acción colectiva, pues "nos interesa reunirnos con las personas que representan las instituciones, para que pueda haber continuidad y trascendencia de lo que se hace. Lo que estamos pensando no es de manera individual, porque uno está hoy, pero no sabe mañana. Es más bien la responsabilidad que tenemos con el país y con los territorios donde estamos”. ${ }^{23}$

Sin embargo, cuando se busca que la Red Prodepaz se pronuncie frente a la realidad nacional surge la pregunta de la representatividad. ¿A quiénes representa la Red? ¿Cuál es la identidad de la Red? E inmediatamente aparece también la pregunta sobre la identidad de los PDP, y se les considera como respuestas regionales a una "sin-salida” que el país tiene hace mucho tiempo. La red cumple un papel de facilitar esos espacios de encuentro entre los PDP de las distintas regiones, pero no es un movimiento social y tampoco es un sujeto actor. Entonces, ¿podemos tipificarla?

La Red partió de la convicción ética sobre el papel y la responsabilidad de la sociedad civil frente a la guerra y la pobreza e incluyó a las iglesias, la empresa privada, las organizaciones sociales y las universidades, entre otras, para ejecutar propuestas de desarrollo y paz frente al conflicto armado que se vive en las regiones. Para impulsar los PDP en red en las regiones, y para que éstos puedan evolucionar de manera positiva y propositiva, es necesaria la presencia de un equipo gestor e impulsor que tenga conocimiento y comprensión del problema social colombiano, o que al menos en su inicio presente una actitud abierta y esté dispuesto a escuchar a los pobladores regionales para construir con ellos la propuesta de desarrollo y paz. "Si no hay equipo formado, no es posible desarrollar procesos de desarrollo y paz”, ${ }^{24}$ El establecimiento de un punto de referencia que cualifique la intervención social en el territorio pasa por un trabajo permanente alrededor del cambio personal e interno que a su vez hace posible un cambio interno en las organizaciones locales y, así mismo, proyectarlas externamente hacia transformaciones regionales. Si bien las propuestas de los PDP son más locales y regionales que centrales, la función de la red de

\footnotetext{
${ }^{22}$ Van Deventer, Fulco, “Comprendiendo las Redes”, octubre de 2003, en: g:licclthemas\netwerken\Netwerken Fulco\Begrip voor Netwerken NL FvD 15 okt0303

${ }^{23}$ Entrevista a representante de ISA, Bogotá, octubre de 2005.

${ }^{24}$ Entrevista a una persona del equipo de Prodepaz. Bogotá, septiembre de 2005.
} 
redes que cumple la Red Prodepaz es fundamental para que los PDP puedan llegar a tener un impacto a escala nacional. Esta labor requiere crear confianza a partir de un balance de las organizaciones locales sin exclusiones, y realizar un esfuerzo para no dejarse polarizar ni manipular por los grupos armados u otro tipo de actores que tengan el mismo objetivo. Puede decirse que es una forma de proteger el trabajo para participar en la construcción de región. Pero crear confianza es un proceso complejo y toma tiempo. Es necesario conocer el territorio, enamorarse de él, vivir en él, identificarse con él, sentir amor por las personas que allí viven, comprender su lógica y sus necesidades e intereses, tener una actitud de diálogo y creer que es posible un cambio, comenzando por el personal.

\section{Reflexiones finales}

La intervención social en los PDP, en la Red Prodepaz y en el CINEP, que forma parte de varios de los $\mathrm{PDP}^{25}$ y de la red Prodepaz, es abordada con una mirada compleja que facilita la comprensión de las redes sociales, que son las que mejor definen nuestra forma de hacer el trabajo. Partimos de ubicarnos en medio de la complejidad de actores, intereses, conflictos, identidades, y desde la aceptación de la diversidad nos situamos como un actor más en las regiones, no como el actor o actriz principal. Es decir reconocemos con simpatía (poniéndonos en los zapatos del otro) a los otros actores de las regiones y a la institucionalidad presente en ellas (alcaldías, gobernaciones, organizaciones de la sociedad civil, organizaciones sociales y populares) y construimos sinergias, hacemos gestión y alianzas estratégicas. Por eso no dejamos por fuera a ningún actor e incluimos a todos los actores del territorio, incluyendo los actores armados, con quienes se dialoga para hacer visibles a las víctimas del conflicto y para promover los derechos a la verdad, la justicia y la reparación que ellas tienen, así como para promover la defensa de los derechos humanos y un modelo de desarrollo incluyente y distinto al actual que acentúa la exclusión y la pobreza. Trabajar en medio del conflicto supone gestionar el riesgo de la propia vida. Y esto supone la capacidad de anticiparse a las amenazas y disminuir las vulnerabilidades personales y del

\footnotetext{
${ }^{25}$ PDPMM, Prodepaz, Cordepaz, Mirada al Sur.
} 
grupo a través de protocolos que suponen conocimiento y práctica de los derechos humanos, conocimiento de cómo funcionan las alertas tempranas, conocimiento de sí mismo, comunicación y responsabilidad por sí mismo. También supone crear lazos de afecto y protección que puedan ayudar a la defensa de la vida.

Partimos del conocimiento y del análisis del contexto de las regiones, conocimiento construido en las interacciones con la gente que vive en ellas. El diálogo y la concertación marcan la pauta de la construcción colectiva. Sabemos que un trabajo que se queda en la región, sin articulaciones con otras regiones y con el país, se agota por su aislamiento y puede llegar a dejar de tener sentido. Por eso la red de redes que es la Red Prodepaz propone generar un espacio de encuentro, análisis de experiencias, gestión de lo público, relaciones con la cooperación internacional y con el Gobierno nacional y, sobretodo, un espacio de propuestas alternativas viables al modelo de intervención social actual centrado muchas veces en políticas de corte asistencialista. Para el equipo del CINEP este modelo de intervención social contiene tres componentes :

1. Análisis de contexto: historia de construcción de identidades y de relaciones entre los pobladores entre sí y con su entorno y territorio, de poblamiento de las regiones, actores-sujetos, intereses y conflictos, posesión y destino de la tierra, presencia de las multinacionales, re-conformación de nuevos poblamientos en los territorios.

2. Marco institucional: el propósito básico nuestro es la apuesta por la vida y la participación en la construcción de una sociedad justa. Esto lo hacemos mediante la construcción de alternativas de desarrollo y paz. Las estrategias que utilizamos son la investigación y sistematización, la gestión y mediación, la producción de información, la educación y la comunicación.

3. Enfoque de desarrollo y paz: la construcción de alternativas de desarrollo y paz se realiza mediante procesos que llamamos centrales y están constituidos por el fortalecimiento del tejido social, el pensamiento complejo y la incidencia en lo público. 
Estos componentes de la intervención social van acompañados de seis criterios que interactúan entre sí:

1. Integralidad: visión compleja que integra las dimensiones que interactúan en el territorio (económica, política, cultural, social, ambiental y ética).

2. Complejidad y diferenciación: trabajamos la construcción social del territorio de manera diferenciada y compleja desde los sujetos (hombres y mujeres) para la transformación de la sociedad.

3. Autonomía de las y los sujetos: potenciamos actores-sujetos autónomos con capacidad para construir propuestas de desarrollo y paz mediante la investigación y la sistematización de experiencias.

4. Mirada prospectiva con criterios de trabajo.

5. Diálogo y concertación: desencadenamos procesos de diálogo, cabildeo, negociación, sinergia y mediación para la construcción del desarrollo y la paz.

6. Sostenibilidad: gestionamos la viabilidad de los procesos sociales, políticos, económicos, ambientales y culturales de los sujetos en los territorios. Se trabaja igualmente por la sostenibilidad financiera de la institución.

\section{Bibliografía}

Angulo, Alejandro, Ética, solidaridad y ecología, CINEP, Bogotá, 1994.

Barcena, Iñaki. “¿De qué hablamos cuando hablamos de desarrollo sostenible? Discursos y políticas ambientales en lid”, en Varios autores. Hacia un desarrollo rural sostenible, Ecos Association, Cantabria, 2001.

Bossier, Sergio, “Crisis y alternativas en los procesos de regionalización”, en: Revista de la CEPAL, No. 52, abril 1994.

CINEP, Seminario de Desarrollo y Paz, 2006. 
Collier, Paúl. "Causas económicas de las guerras civiles y sus implicaciones para el diseño de políticas”, en: El Malpensante, No. 30, 2001.

Cuervo, Jorge Iván, “La gestión de lo público más allá de lo estatal: el caso del Fondo de Reconstrucción Social y Económica del Eje Cafetero,(FOREC), Colombia”. Tesis de grado, Universidad de Chile, 2001.

De Roux, Francisco, "Documento central de diagnóstico, conclusiones y recomendaciones, Programa de Desarrollo y Paz en el Magdalena Medio, PDPMM”, Consorcio SEAP-CINEP, Mimeografía, Bogotá,1996.

Fals Borda, Orlando, Región e historia: elementos sobre ordenamiento y equilibrio regional en Colombia, Tercer Mundo Editores, Bogotá, 1996.

García, Arturo y Alfredo Sarmiento (coordinadores), Programas regionales de desarrollo y paz: Casos de capital social y desarrollo institucional. Fundación Ideas Para la Paz- PNUD, Bogotá, 2002.

González, Fernán, “El conflicto, callejón con salida”, en: Informe nacional de desarrollo humano, Colombia, Naciones Unidas, 2003.

González, Fernán, Teófilo Vásquez e Ingrid Bolívar, Violencia Política en Colombia: de la nación fragmentada a la construcción del Estado, CINEP, Bogotá, 2003

González, Jorge Iván, y otros, “Evaluación Externa del segundo crédito de aprendizaje e innovación (LIL II) del Programa de desarrollo y Paz del Magdalena Medio”, Centro de Investigaciones del Desarrollo (CID), Universidad Nacional de Colombia, noviembre 2003.

González, Jorge Iván, Imaginarios de Desarrollo y Paz en el Magdalena Medio, CINEP, 2003

González, Jorge Iván, “Bien-Estar: Macroeconomía y Pobreza”, Informe de Coyuntura, Contraloría General de la República - Centro de Investigaciones del Desarrollo (CID), Universidad Nacional de Colombia, Bogotá, 2004.

Hernández, Esperanza, "La paz y la no violencia adquieren significado propio en Colombia en las iniciativas de paz que construyen las bases desde lo local”, en: Reflexión Política, Año 4, No. 8, diciembre de 2002, (p 106-116).

Honewey, Martin, "Redprodepaz, The Organizational Development and Learning Programmer, Developing Organizational Infrastructure in Zones of Conflict”, documento inédito, Bogotá, 2005.

Laboratorio de Paz del Magdalena Medio, Documento Central, en: http://www.pdpmm.org.co/docs/fichas/a_0013.doc 
Machado, Absalón, "Seguridad alimentaria y sistema agro-alimentario" en: Revista Univerciudad, Número 15, 2005. Documento completo en PDF en "Programa de Bogotá sin hambre”.

Max-Neef, Manfred, Desarrollo a escala humana: una opción para el futuro, Nueva Editorial, Santiago de Chile, 2000.

Otsberg, Sonny, Documento Interno, 2005.

Red Prodepaz, “Informe Anual de Gestión”, Asamblea General, noviembre de 2004.

Red Prodepaz, "Los Programas de Desarrollo y Paz: un esfuerzo del desarrollo local en medio del conflicto”, 2004

Red Prodepaz, “Declaración de marzo de 2002”.

Rincón, Juliet, “Documentos internos de la Red Prodepaz”, 2003.

Saavedra, María del Rosario y Diego Luís Ojeda, “Trabajo en Red. Imaginarios conceptuales de Desarrollo, Paz y Región”, CINEP, julio de 2006.

Stiglitz, Joseph, El malestar en la globalización, Taurus, Madrid, 2002.

Sen, Amartya, Desarrollo y Libertad, Editorial Planeta, Bogotá, 2003.

Touraine, Alain, Critique de la Modernité, Ediciones Fayard, Paris, 1992.

Touraine, Alain, “¿Podremos vivir Juntos? El destino del hombre en la aldea global, Fondo de Cultura Económica, Buenos Aires, 1996.

Touraine, Alain y Farhad Khosrokhavar, A la búsqueda de sí mismo. Diálogo sobre el sujeto, Paidós, Buenos Aires, 2002.

Van Deventer, Fulco, “Comprendiendo las Redes”, octubre de 2003, en: g: Iicclthemas\netwerken \Netwerken Fulco\Begrip voor Netwerken NL FvD 15 okt0303

Vargas, Marcos y Alberto Rincón, “Los Rostros del Magdalena Medio”, CDPMM-CINEP, Bogotá, Mimeo, 2001

http://www.opi.org.co/Culturadepaz.htm

http://www.pastoralsocialcolombia.org/imagenescaritas/Eventos/congreso-archivo.rtf

http://www.nasaacin.net/historia_general.htm 
http://www.ideaspaz.org/proyecto04/programas_regionales_desarrollo_paz.pdf 